\title{
Interdependence of different symmetry energy elements
}

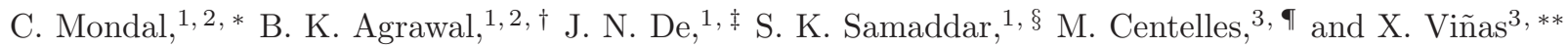 \\ ${ }^{1}$ Saha Institute of Nuclear Physics, 1/AF Bidhannagar, Kolkata 700064, India \\ ${ }^{2}$ Homi Bhabha National Institute, Anushakti Nagar, Mumbai 400094, India. \\ ${ }^{3}$ Departament de Fúsica Quàntica i Astrofísica and Institut de Ciències del Cosmos (ICCUB), \\ Facultat de Física, Universitat de Barcelona, Martí i Franquès 1, E-08028 Barcelona, Spain
}

(Dated: October 15, 2018)

\begin{abstract}
Relations between the nuclear symmetry energy coefficient and its density derivatives are derived. The relations hold for a class of interactions with quadratic momentum dependence and a power-law density dependence. The structural connection between the different symmetry energy elements as obtained seems to be followed by almost all reasonable nuclear energy density functionals, both relativistic and non-relativistic, suggesting a universality in the correlation structure. This, coupled with known values of some well-accepted constants related to nuclear matter, helps in constraining values of different density derivatives of the nuclear symmetry energy shedding light on the isovector part of the nuclear interaction.
\end{abstract}

The nuclear symmetry energy elements encode in them imprints of the nature of the isovector part of the nuclear interaction. They have profound implications not only in terrestrial nuclear physics but also in astrophysics and cosmology [1 3$]$. Some of them like the symmetry energy coefficient $C_{2}(\rho)\left(=\frac{1}{2}\left(\frac{\partial^{2} e(\rho, \delta)}{\partial \delta^{2}}\right)_{\delta=0}\right.$, where $e(\rho, \delta)$ is the energy per nucleon of nuclear matter of density $\rho$ at isospin asymmetry $\delta$ ) is now known in tighter bounds at the saturation density $\rho_{0}$ [4, 5] of symmetric nuclear matter (SNM). From analysis of the giant dipole resonance (GDR) of ${ }^{208} \mathrm{~Pb}$, a well-constrained estimate of $C_{2}(\rho)$ at a somewhat lesser density $(\rho=0.1$ $\mathrm{fm}^{-3}$ ) [6] is also known. The value of the density slope of the symmetry energy $L_{0}\left(=L\left(\rho_{0}\right)=3 \rho_{0}\left(\frac{\partial C_{2}}{\partial \rho}\right)_{\rho_{0}}\right)$ is less certain [3, 7, 8]. The shroud of uncertainty looms even larger on the higher derivatives of the symmetry energy [e.g., $K_{\text {sym }}^{0}\left(=K_{\text {sym }}\left(\rho_{0}\right)=9 \rho_{0}^{2}\left(\frac{\partial^{2} C_{2}}{\partial \rho^{2}}\right)_{\rho_{0}}\right)$ or $Q_{s y m}^{0}$ $\left.\left(=Q_{\text {sym }}\left(\rho_{0}\right)=27 \rho_{0}^{3}\left(\frac{\partial^{3} C_{2}}{\partial \rho^{3}}\right)_{\rho_{0}}\right)\right]$ and on the difference between the neutron and proton effective masses $\Delta m_{0}^{*}$ $\left[=\left(m_{n}^{*}-m_{p}^{*}\right) / m\right]$ in neutron-rich matter at $\rho_{0}$. The values of $K_{\text {sym }}^{0}$ and $Q_{\text {sym }}^{0}$, in different parametrizations of the Skyrme energy density functional (EDF) lie in very wide ranges $\left[-700 \mathrm{MeV}<K_{\text {sym }}^{0}<400 \mathrm{MeV} ;-800 \mathrm{MeV}\right.$ $\left.<Q_{\text {sym }}^{0}<1500 \mathrm{MeV}\right]$ [9, 10] whereas there are divergent predictions on the value of $\Delta m_{0}^{*}$ from theoretical studies based on microscopic many-body theories [11, 12] or phenomenological approaches 13 16. Such large uncertainties belie a satisfactory understanding of the nuclear isovector interaction.

\footnotetext{
*Electronic address: chiranjib.mondal@saha.ac.in

$\dagger$ Electronic address: bijay.agrawal@saha.ac.in

$\ddagger$ Electronic address: jn.de@saha.ac.in

$\S$ Electronic address: santosh.samaddar@saha.ac.in

ๆ Electronic address: mariocentelles@ub.edu

**Electronic address: xvinasg@ub.edu
}

There is a sliver of expectation that the entities $C_{2}^{0}$ $\left(=C_{2}\left(\rho_{0}\right)\right), L_{0}, K_{\text {sym }}^{0}$, etc. may have an intrinsic correlation among them. Finding a correlated structure for these symmetry energy elements helps in making a somewhat more precision statement on an otherwise uncertain isovector indicator as it may be tied up to another one known with more certainty. There is a hint of a relatively weak positive correlation between $C_{2}^{0}$ and $L_{0}$ [9, 10]. From observation of the computed values of $L_{0}$ and $K_{\text {sym }}^{0}$ with selected sets of non-relativistic and relativistic EDFs, a fairly linear relationship between $K_{\text {sym }}^{0}$ and $L_{0}$ is also suggested [15, 17-22]. The present communication aims to point to some possible universal aspects of the nuclear EDFs related to nuclear symmetry energy elements. As we will see, in a general mean-field approach analytic relationships can be built up among the nuclear symmetry energy elements tieing them in a correlated structure and helping in finding their values in terms of a few empirical nuclear constants.

For symmetric nuclear matter at density $\rho$, with energy density $\mathcal{H}$, and at zero temperature $(T=0)$, the chemical potential of the nucleon is given by

$$
\mu=\mathcal{E}_{F}=\frac{P_{F}^{2}}{2 m^{*}}+V=\frac{P_{F}^{2}}{2 m}+U,
$$

where $\mathcal{E}_{F}$ is the Fermi energy, $P_{F}$ is the Fermi momentum, and the effective mass $m^{*}$ and the single-particle potential $V$ are given, respectively, by $\hbar^{2} / 2 m^{*}=\delta \mathcal{H} / \delta \mathcal{K}$ and $V=\delta \mathcal{H} / \delta \rho$, where $\frac{\hbar^{2}}{2 m} \mathcal{K}$ is the kinetic energy density. One also can redefine the single-particle potential as $U$ by including within it the effective mass contribution, as done in the r.h.s. of Eq. (11). We make no special assumption about the nucleonic interaction except that it is density dependent to simulate many-body forces and that it depends quadratically on the momentum; thus, the single-particle potential $U$ separates into three parts,

$$
U=V_{0}+P_{F}^{2} V_{1}+V_{2} .
$$

The term $\left(V_{0}+P_{F}^{2} V_{1}\right)$ on the right is the Hartree-Fock potential and the last term $V_{2}$ is the rearrangement potential that arises from the density dependence in the 
interaction. The term $V_{1}$ comes from the momentum dependence:

$$
\frac{P_{F}^{2}}{2 m^{*}}=\frac{P_{F}^{2}}{2 m}+P_{F}^{2} V_{1} .
$$

In general, $m^{*}$ is momentum and energy dependent, in the mean-field level the energy dependence is ignored and the momentum dependence is taken at the Fermi surface. The rearrangement energy does not enter explicitly in the energy expression when written in terms of the meanfield potential [23, 24], the energy per nucleon for SNM at density $\rho$ is then given by,

$$
\begin{aligned}
e & =\frac{1}{2 m}\left\langle p^{2}\right\rangle+\frac{1}{2}\left\langle p^{2}\right\rangle V_{1}+\frac{1}{2} V_{0} \\
& =\frac{1}{4}\left(\frac{1}{m}+\frac{1}{m^{*}}\right)\left\langle p^{2}\right\rangle+\frac{1}{2} V_{0}
\end{aligned}
$$

The Gibbs-Duhem relation relates the chemical potential and energy as,

$$
\mu=e+\frac{P}{\rho}
$$

where $P$ is the pressure of the system. At zero pressure this leads to the Hugenholtz-Van Hove theorem 25] which has recently been used to link nucleon singleparticle characteristics to macroscopic isovector properties in Ref. [26]. Keeping this in mind, from Eqs. (11), (44) and (5), the energy per nucleon for SNM can be written as [27],

$$
e=\frac{P_{F}^{2}}{10 m}\left(3-2 \frac{m}{m^{*}}\right)-V_{2}+\frac{P}{\rho},
$$

where $\left\langle p^{2}\right\rangle=3 P_{F}^{2} / 5$ has been used.

For asymmetric nuclear matter, the equation for the energy per nucleon can be generalized as

$$
\begin{aligned}
e(\rho, \delta)= & \frac{1}{\rho}\left[\sum_{\tau} \frac{P_{F, \tau}^{2}}{10 m} \rho_{\tau}\left(3-2 \frac{m}{m_{\tau}^{*}(\rho)}\right)\right]-V_{2}(\rho, \delta) \\
& +\frac{P(\rho, \delta)}{\rho}
\end{aligned}
$$

In Eq.(7), $\tau$ is the isospin index, $\rho_{\tau}=(1+\tau \delta) \rho / 2$; here, $\tau=1$ for neutrons and $\tau=-1$ for protons. The Fermi momentum for the individual species can be written as $P_{F, \tau}=g_{2} \rho_{\tau}^{1 / 3}$ with $g_{2}=\left(3 \pi^{2}\right)^{1 / 3} \hbar$. The densitydependent nucleon effective mass is written as

$$
\frac{m}{m_{\tau}^{*}(\rho)}=1+\frac{k_{+}}{2} \rho+\frac{k_{-}}{2} \rho \tau \delta .
$$

This is a generalization from $\frac{m}{m^{*}}=1+k \rho$, usually taken in a nonrelativistic prescription for SNM [28]. The density dependence in the rearrangement potential is taken as

$$
V_{2}(\rho, \delta)=\left(a+b \delta^{2}\right) \rho^{\tilde{\alpha}}
$$

which is independent of the isospin index $\tau$. The constant $a$ weighs the rearrangement potential for SNM, whereas the constant $b$ is a measure of the asymmetry dependence of the rearrangement potential.

The energy per nucleon $e(\rho, \delta)$ can also be written in terms of the symmetry energy coefficients as

$$
e(\rho, \delta)=e(\rho, \delta=0)+C_{2}(\rho) \delta^{2}+C_{4}(\rho) \delta^{4}+\cdots
$$

The expression for the pressure $P(\rho, \delta)=\rho^{2} \frac{\partial e}{\partial \rho}$ follows from the above equation. Hence, the right hand side of Eq.(7) can be expanded in powers of $\delta$ using the expressions for $P(\rho, \delta)$ and $V_{2}(\rho, \delta)$ and using Eq.(8). Comparing then with Eq.(10) and equating coefficients of the same order in $\delta$, one gets the expression for $C_{2}(\rho)$ as

$$
\begin{aligned}
C_{2}(\rho)= & -b \rho^{\tilde{\alpha}}+\rho \frac{\partial C_{2}(\rho)}{\partial \rho}+y \rho^{2 / 3}\left[-\frac{5}{3} k_{-} \rho\right. \\
& \left.+\frac{5}{9}\left(1-k_{+} \rho\right)\right]
\end{aligned}
$$

with $y=\frac{g_{2}^{2}}{10 m} \frac{1}{2^{2 / 3}}$. The relation between $C_{2}(\rho)$ and its density derivative is a direct consequence of the GibbsDuhem relation. At saturation density the symmetry energy coefficient $C_{2}$ reads as,

$$
\begin{aligned}
C_{2}^{0}= & -b \rho_{0}^{\tilde{\alpha}}+\frac{L_{0}}{3}+E_{F}^{0}\left[-\frac{1}{3} k_{-} \rho_{0}\right. \\
& \left.+\frac{1}{9}\left(1-k_{+} \rho_{0}\right)\right],
\end{aligned}
$$

where $E_{F}^{0}=5 y \rho_{0}^{2 / 3}$ is the Fermi energy at $\rho_{0}$. Similar equations can be obtained for higher-order symmetry energy coefficients $C_{4}, C_{6}$, etc. which we do not deal here. The expressions for $C_{2}$ or the higher-order symmetry energy coefficients so obtained are exact within the precincts of the premises we have chosen. Taking second and third density derivatives of $C_{2}(\rho)$ in (11), expressions for $K_{s y m}^{0}$ and $Q_{\text {sym }}^{0}$ at saturation can then be obtained, which with the help of Eq. (12) read

$$
\begin{aligned}
K_{\text {sym }}^{0}= & -3 \tilde{\alpha}\left[3 C_{2}^{0}-L_{0}\right]+E_{F}^{0}[(3 \tilde{\alpha}-4) \\
& \left.+\left(\frac{2}{3} \frac{m}{m_{0}^{*}}+k_{-} \rho_{0}\right)(5-3 \tilde{\alpha})\right]
\end{aligned}
$$

and

$$
\begin{aligned}
Q_{\text {sym }}^{0}= & 15 \tilde{\alpha}\left[3 C_{2}^{0}-L_{0}\right]+K_{\text {sym }}^{0}(3 \tilde{\alpha}-1) \\
& +E_{F}^{0}(2-3 \tilde{\alpha})
\end{aligned}
$$

While exploring the standard Skyrme EDFs, we found that exactly this correlated structure of $K_{\text {sym }}^{0}$ or $Q_{s y m}^{0}$ with $\left[3 C_{2}^{0}-L_{0}\right]$ as in Eqs. (13) and (14) is obtained.

Eq. (13) throws a hint that there is a strong likelihood that $K_{\text {sym }}^{0}$ calculated with different EDFs may be linearly correlated to $\left[3 C_{2}^{0}-L_{0}\right]$ corresponding to the EDFs. This is vindicated from the correlated structure of $K_{s y m}^{0}$ 


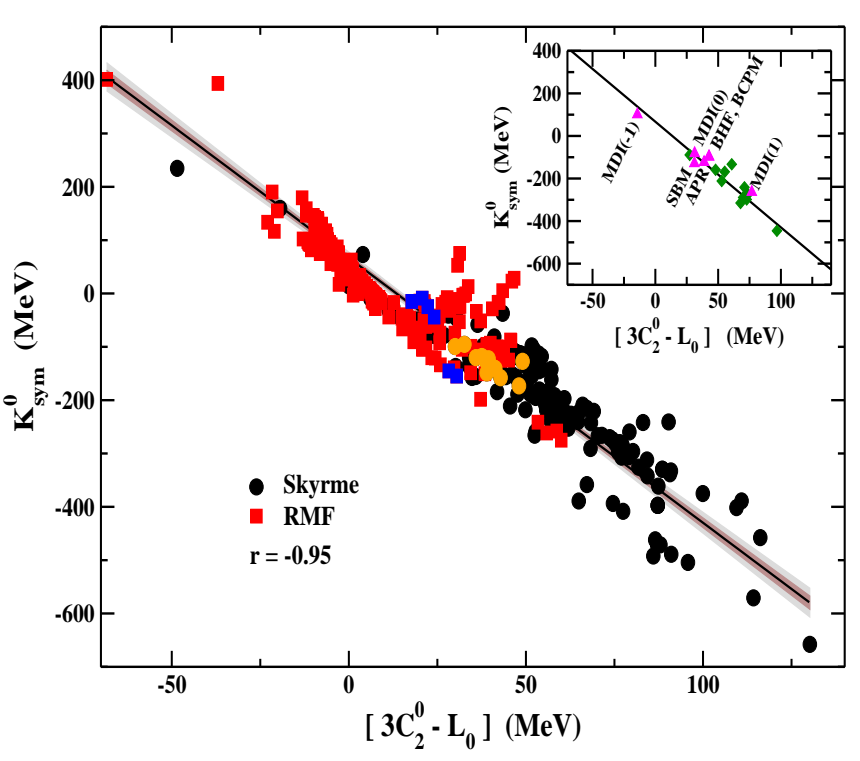

FIG. 1: (Color online) The correlation between $K_{\text {sum }}^{0}$ and $\left[3 C_{2}^{0}-L_{0}\right]$ as obtained from five hundred EDFs [9, [10]. The black circles correspond to the Skyrme-inspired EDFs, the red squares refer to those obtained from RMF models. The models consistent with all the constraints demanded by Dutra et al. are highlighted by orange circles for Skyrme EDFs [9] and blue squares for RMF EDFs [10]. The inner (outer) colored regions around the best-fit straight line through these points depict the loci of $95 \%$ confidence (prediction) bands of the regression analysis. The inset shows the correlation line obtained from the Skyrme-RMF models, the magenta triangles are the results obtained from EDFs with realistic interactions, MDI(0), MDI(1), MDI(-1) 15], APR 29], BHF 30], BCPM 31] and SBM [32], respectively. The green diamonds represent results from a few Gogny interactions [14].

with $\left[3 C_{2}^{0}-L_{0}\right]$ as displayed in Fig 1 for five hundred energy density functionals [9, 10] that have been in use to explain nuclear properties. The results as presented in Fig. 1 span both the Skyrme-inspired nonrelativistic (black circles) EDFs which tend to have negative values for $K_{\text {sym }}^{0}$ and also the relativistic mean-field EDFs (red squares) that tend to have larger, sometimes positive values for $K_{\text {sym }}^{0}$. We highlight those Skyrme (orange circles) and RMF (blue squares) models chosen by Dutra et. al. [9, 10] which were found to satisfy specific constraints on nuclear matter and neutron star properties. The linear correlation as observed seems to be nearly universal and intrinsic to an EDF consistent with nuclear properties. The correlation coefficient $(r)$ is seen to be $r=-0.95$. The near-universality in the correlation is brought into sharper focus in the inset in the figure where results corresponding to EDFs obtained from several realistic interactions (magenta triangles) and a few Gogny interactions (green diamonds) are displayed and are seen to lie nearly on the correlation line. The linear regression analysis yields

$$
K_{\text {sym }}^{0}=d_{1}\left[3 C_{2}^{0}-L_{0}\right]+d_{2},
$$

with $d_{1}=-4.97 \pm 0.07$ and $d_{2}=66.80 \pm 2.14 \mathrm{MeV}$. This is a robust correlation among the symmetry energy elements. Incidentally, from the density-dependent M3Y (DDM3Y) interaction, a similar kind of relation between these symmetry elements can be observed [21]. The correlation between the $K_{\text {sym }}^{0}$ and $L_{0}$ values from different effective forces and realistic interactions has also been considered in previous literature [15, 17 -22]. The results have shown relatively varying degrees of correlation. In our case, the correlation between $K_{\text {sym }}^{0}$ and $L_{0}$ from all the five hundred EDFs of Fig. 1 is not found to be as strong as the correlated structure of $K_{\text {sym }}^{0}$ with $\left[3 C_{2}^{0}-L_{0}\right]$, the correlation coefficient between $K_{\text {sym }}^{0}$ and $L_{0}$ is seen to be $r=0.87$. The correlation between $Q_{s y m}^{0}$ with $\left(3 C_{2}^{0}-L_{0}\right)$ is incidentally not as good as for $K_{\text {sym }}^{0}$, the correlation coefficient is 0.66 . This is possibly because of propagation of errors from $K_{\text {sym }}^{0}$.

From accumulated experimental data over several decades and their theoretical analyses, there seems to be a broad consensus about the values of some of the nuclear constants. The saturation density $\rho_{0}$ of SNM, its energy per nucleon $e_{0}$ and its incompressibility coefficient $K_{0}$ are taken as a subset of the constants characterizing symmetric nuclear matter. The nucleon effective mass $m_{0}^{*}$ for SNM at $\rho_{0}$ is also taken as an input datum though its value is not as certain as $e_{0}$ or $\rho_{0}$. Two more nuclear constants related to asymmetric nuclear matter (ANM) are further considered. They are the nuclear symmetry energy coefficients $C_{2}(\rho)$ at $\rho_{0}$ and at a somewhat lesser density $\rho_{1}\left(=0.1 \mathrm{fm}^{-3}\right)$, "the crossing density". There is less room for uncertainty in the symmetry energy coefficient $C_{2}^{0}$ which has been determined from exploration of nuclear masses [4, 5]. With the realization that the nuclear observables related to average properties of nuclei constrain the nuclear EDFs better at around the average density of terrestrial atomic nuclei 33], the so-called "crossing density" 34 assumes a special significance. The symmetry energy $C_{2}^{1}\left(=C_{2}\left(\rho_{1}\right)\right)$ at that density, in Skyrme EDFs is seen to be strongly correlated to the Giant Dipole Resonance (GDR) in spherical nuclei and is now fairly well constrained [6]. From the apparently universal, EDF-independent correlation between the isovector observables, the isovector elements $L_{0}, K_{\text {sym }}^{0}$, etc. can now be threaded to the above-mentioned nuclear constants as we show below.

With $m_{0}^{*}$ as input, $k_{+}$is known. From given values of $e_{0}, \rho_{0}$ and $K_{0}$ for SNM, $\tilde{\alpha}$ can be calculated as [27],

$$
\tilde{\alpha}=\frac{\frac{K_{0}}{9}+\frac{E_{F}^{0}}{3}\left(\frac{12}{5}-2 \frac{m}{m_{0}^{*}}\right)}{\frac{E_{F}^{0}}{5}\left(3-2 \frac{m}{m_{0}^{*}}\right)-e_{0}} .
$$

The symmetry energy $C_{2}\left(\rho_{1}\right)$ can be expressed as

$$
C_{2}\left(\rho_{1}\right)=C_{2}^{0}-L_{0} \epsilon+\frac{1}{2} K_{\text {sym }}^{0} \epsilon^{2}
$$




$$
-\frac{1}{6} Q_{s y m}^{0} \epsilon^{3}+\cdots
$$

where $\epsilon=\frac{\left(\rho_{0}-\rho_{1}\right)}{3 \rho_{0}}$. From Eqs. (14), (15) and (17), ignoring terms beyond $\epsilon^{3}$, which are negligible, $L_{0}, K_{\text {sym }}^{0}$ and $Q_{\text {sym }}^{0}$ are calculated with known values of $C_{2}^{0}$ and $C_{2}^{1}$. The constant $k_{-}$then follows from Eq. (13). From Eq. (8), the nucleon effective mass splitting at saturation density to leading order in $\delta$ is given as

$$
\Delta m_{0}^{*}=\left(\frac{m_{n}^{*}-m_{p}^{*}}{m}\right)_{\rho_{0}} \simeq-k_{-} \rho_{0}\left(\frac{m_{0}^{*}}{m}\right)^{2} \delta
$$

where the approximation $\left(m_{n}^{*} \cdot m_{p}^{*}\right) \simeq\left(m_{0}^{*}\right)^{2}$ is made.

Comparing Eqs. (13) and (15) one would expect $\left|d_{1}\right|$ to be close to $3 \tilde{\alpha}$. With the input values of the isoscalar nuclear constants $e_{0}, \rho_{0}$ and $K_{0}, 3 \tilde{\alpha}$ is seen to be 3.54 as opposed to $\sim 5$ for $\left|d_{1}\right|$. The reason for this change seems to be two-fold, (a) all 500 EDFs employed in Fig. 11 have different values for $\tilde{\alpha}$, and (b) the RMF models are also included in the fit which have no explicit counterpart of $\tilde{\alpha}$.

In summary, the values of $L_{0}, K_{\text {sym }}^{0}, Q_{\text {sym }}^{0}$ and $\Delta m_{0}^{*}$ can be calculated in terms of empirically known nuclear constants namely, $\rho_{0}, e_{0}, K_{0}, C_{2}^{0}, C_{2}^{1}$ and $\frac{m_{0}^{*}}{m}$ using Eqs. (14)-(18). From the diverse theoretical endeavours like the liquid drop type models [4, 35, 36], the microscopic ab-initio or variational calculations [31, 37], or different Skyrme or RMF models - all initiated to explain varied experimental data, a representative set of the input nuclear constants for SNM is chosen with $\rho_{0}=0.155 \pm 0.008 \mathrm{fm}^{-3}$ and $e_{0}=-16.0 \pm 0.2 \mathrm{MeV}$. From microscopic analysis of isoscalar giant monopole resonances (ISGMR), the value of $K_{0}$ is constrained as $230 \pm 40 \mathrm{MeV}$ [33]. Analyzing the compact correlation between the 'experimental' double-differences of symmetry energies of finite nuclei and their mass number, Jiang et. al. [5] find $C_{2}^{0}=32.1 \pm 0.3 \mathrm{MeV}$. We include this value in our set of nuclear constants. For $C_{2}^{1}$, the value $C_{2}^{1}=24.1 \pm 0.8 \mathrm{MeV}$ as quoted from microscopic analysis of GDR in ${ }^{208} \mathrm{~Pb}$ [6] is taken. There is an overall consistency of this $C_{2}^{1}$ value with those from the best-fit Skyrme EDFs 34] and with that given in 21]. For the nucleon effective mass, a value of $\frac{m_{0}^{*}}{m}=0.70 \pm 0.05$ is taken, this is consistent with the empirical values obtained from many analyses [38, 39].

The values of the symmetry energy elements calculated from Eqs. (14) - 18) using the values of input nuclear constants as mentioned come out to be $L_{0}=60.3 \pm 14.5$ $\mathrm{MeV}, K_{\text {sym }}^{0}=-111.8 \pm 71.3 \mathrm{MeV}, Q_{\text {sym }}^{0}=296.8 \pm 73.6$ $\mathrm{MeV}$ and $\Delta m_{0}^{*}=(0.17 \pm 0.24) \delta$. The value of $L_{0}$ is remarkably close to its global average $58.9 \pm 16 \mathrm{MeV}$ [40], obtained from analyses of terrestrial experiments and astrophysical observations. The value of $L$ at $\rho_{1}$ is calculated to be $49.3 \pm 4.2 \mathrm{MeV}$. From dipole polarizability in ${ }^{208} \mathrm{~Pb}$ an empirical value of $L=47.3 \pm 7.8 \mathrm{MeV}$ was obtained at $\rho \simeq 0.11 \mathrm{fm}^{-3}$ [1]. To our knowledge, there is no experimental value for $K_{s y m}^{0}$ or $Q_{s y m}^{0}$ to compare.
However, the symmetry incompressibility $K_{\delta}$ defined at the saturation density of nuclear matter at asymmetry $\delta$ $\left(K_{\delta}=K_{s y m}^{0}-6 L_{0}-\frac{Q_{0} L_{0}}{K_{0}}\right.$, where $\left.Q_{0}=27 \rho_{0}^{3}\left(\frac{\partial^{3} e}{\partial \rho^{3}}\right)_{\rho_{0}}\right)$ has been extracted from breathing mode energies of Snisotopes [42]. Corrected for the nuclear surface term, $K_{\delta}$ is quoted to be $\simeq-350 \mathrm{MeV}$ [43]. This is in close agreement with our calculated value $K_{\delta}=-378.6 \pm 17.0 \mathrm{MeV}$; $Q_{0}$ has been calculated from Eq. (17) to be $-364.7 \pm 27.7$ $\mathrm{MeV}$ corresponding to $\delta=0$ [27] with the input nuclear constants mentioned. Experimental search for $\Delta m_{0}^{*}$, till now is scanty and not very certain. From analysis of nucleon-nucleus scattering data within an isospin dependent optical model [39] it is reported to be $(0.41 \pm 0.15) \delta$. From exploration of isoscalar giant quadrupole resonance and dipole polarizability [4] in ${ }^{208} \mathrm{~Pb}$ it goes down to $(0.27 \pm 0.15) \delta$. Our calculated value for $\Delta m_{0}^{*}$ is on the smaller side. In a recent dynamical BUU calculation [16] with improved isospin and momentum dependent interaction where the isovector giant dipole resonance properties of ${ }^{208} \mathrm{~Pb}$ were used to constrain the nuclear symmetry energy slope parameter and the isospin splitting of the nucleon effective mass, $\delta m_{0}^{*}$ comes close to our value. As a test of the viability of the methodology we have used in this communication, we also calculated $L_{0}, K_{s y m}^{0}$ and $Q_{\text {sym }}^{0}$ for the 16 Skyrme models selected through several tests by Dutra et. al. 9] using their model values of $\rho_{0}$, $e_{0}, K_{0}, C_{2}^{0}, C_{2}^{1}$ and $\frac{m_{0}^{*}}{m}$ as inputs. The so-obtained values of $L_{0}, K_{\text {sym }}^{0}$ and $Q_{\text {sym }}^{0}$ come out to be pretty close to their model values given in Ref. [9] with an average root mean square deviation of $1.3 \mathrm{MeV}$ in $L_{0}, 21.4 \mathrm{MeV}$ in $K_{\text {sym }}^{0}$ and $32.5 \mathrm{MeV}$ in $Q_{\text {sym }}^{0}$, showing the reasonableness of our adopted method.

The set of nuclear constants we have chosen is a conservative set; depending on possible new experimental inputs, their values may however change somewhat which would affect the calculated values of the density derivatives of the symmetry energy coefficients. The evaluated isovector elements are seen to be quite sensitive to the input quantities $C_{2}^{0}, C_{2}^{1}$ and $\rho_{0}$. There is still some variance in the choice of these input nuclear constants [45 47] besides the ones we have chosen. The aforesaid sensitivity can be gauged from the displayed six panels in Fig. 2. In the upper four panels (a)-(d), the contours of constant $L_{0}, K_{\text {sym }}^{0}, Q_{\text {sym }}^{0}$ and $\Delta m_{0}^{*}$ are shown in the $C_{2}^{0}-C_{2}^{1}$ plane in color shades, the white lines within the panels are the loci of constant isovector elements as marked when all other input elements are left unchanged. With increase in $C_{2}^{1}, L_{0}$ and $K_{\text {sym }}^{0}$ are seen to decrease whereas $Q_{\text {sym }}^{0}$ and $\Delta m_{0}^{*}$ are found to increase. The opposite is observed for an increase in $C_{2}^{0}$. This points out the interdependence between different symmetry energy elements. The change in $\rho_{0}$ has also a sizeable effect on the isovector elements. All other inputs remaining intact, an increase in $\rho_{0}$ decreases $L_{0}$ and $K_{\text {sym }}^{0}$ and increases $Q_{\text {sym }}^{0}$ and $\Delta m_{0}^{*}$. We show only glimpses of these changes in panels (e) and (f), where contours of constant $L_{0}$ and $\Delta m_{0}^{*}$ are drawn in the $\rho_{0}-C_{2}^{1}$ plane. The isovector elements as studied 

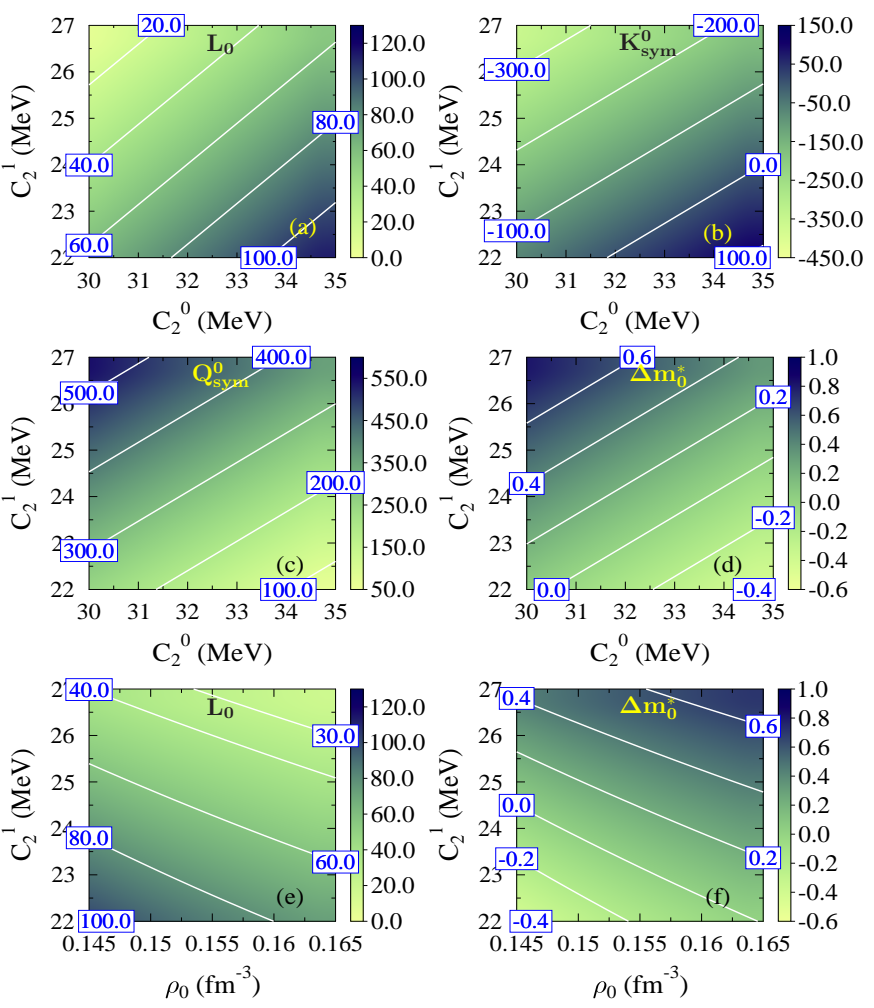

FIG. 2: (Color online) Contours of constant $L_{0}, K_{\text {sym }}^{0}, Q_{\text {sym }}^{0}$ and $\Delta m_{0}^{*}$ in color shades (as indicated on the right side of each panel) as functions of the input nuclear constants $C_{2}^{0}, C_{2}^{1}$ and $\rho_{0}$ depicting the interdependence between various symmetry energy elements. The values of $L_{0}, K_{\text {sym }}^{0}$ and $Q_{\text {sym }}^{0}$ are in units of $\mathrm{MeV}$ and those for $\Delta m_{0}^{*}$ are in units of the free nucleon mass. For details, see text.

here are seen to be nearly insensitive to changes in $e_{0}$ and $m_{0}^{*}$ (not shown here). Similarly, $K_{0}$ has little effect on these isovector elements except on $\Delta m_{0}^{*}$. An increase of $K_{0}$ by, e.g., $\sim 30 \mathrm{MeV}$ is seen to push $\Delta m_{0}^{*}$ drastically in the negative domain. Uncertainties in the input nuclear constants bear signature on the uncertainties in the calculated isovector elements.

To sum up, without reference to any specific nuclear interaction, with only a few reasonable approximations, analytic expressions for the density derivatives of the symmetry energy coefficient $C_{2}(\rho)$ at the saturation density in terms of empirical nuclear constants are found out. The symmetry observables are seen to be sensitive to the values of the input nuclear constants, particularly to $C_{2}^{0}$, $C_{2}^{1}$ and $\rho_{0}$; precise values of these constants are thus required to narrow down the uncertainties in the density dependence of the symmetry energy. In doing the calculations, a correlated structure connecting the different symmetry energy elements emerged. The consonance of these structures with those inherent in the plethora of EDFs of different genre used in nuclear microscopy is very revealing. This indicates a universality in the correlated structure in the symmetry energy coefficients and helps in a better realization of the information content of the isovector observables.

\section{Acknowledgments}

J.N.D. is thankful to the Department of Science and Technology, Government of India for support with the Grant EMR/2016/001512. M.C. and X.V. acknowledge support from Grant FIS2014-54672-P from MINECO and FEDER, Grant 2014SGR-401 from Generalitat de Catalunya, and the project MDM-2014-0369 of ICCUB (Unidad de Excelencia María de Maeztu) from MINECO.
[1] P. J. Woods and C. N. Davids, Annual Review of Nuclear and Particle Science 47, 541 (1997).

[2] J. M. Lattimer and M. Prakash, Phys. Rep. 442, 109 (2007).

[3] B.-A. Li and L.-W. Chen, Modern Physics Letters A 30, 1530010 (2015).

[4] P. Möller, W. D. Myers, H. Sagawa, and S. Yoshida, Phys. Rev. Lett 108, 052501 (2012).

[5] H. Jiang, G. J. Fu, Y. M. Zhao, and A. Arima, Phys. Rev. C 85, 024301 (2012).

[6] L. Trippa, G. Colò, and E. Vigezzi, Phys. Rev. C 77, 061304(R) (2008).

[7] M. Centelles, X. Roca-Maza, X. Viñas, and M. Warda, Phys. Rev. Lett. 102, 122502 (2009).

[8] B. K. Agrawal, J. N. De, and S. K. Samaddar, Phys. Rev. Lett. 109, 262501 (2012).

[9] M. Dutra, O. Lourenço, J. S. Sá Martins, A. Delfino, J. R. Stone, and P. D. Stevenson, Phys. Rev. C 85, 035201 (2012).

[10] M. Dutra, O. Lourenço, S. S. Avancini, B. V. Carlson, A. Delfino, D. P. Menezes, C. Providência, S. Typel, and
J. R. Stone, Phys. Rev. C 90, 055203 (2014).

[11] W. Zuo, L. G. Cao, B. A. Li, U. Lombardo, and C. W. Shen, Phys. Rev. C 72, 014005 (2005).

[12] E. N. E. van Dalen, C. Fuchs, and A. Faessler, Phys. Rev. Lett. 95, 022302 (2005).

[13] L. Ou, Z. Li, Y. Zhang, and M. Liu, Phys. Lett. B 697, 246 (2011).

[14] R. Sellahewa and A. Rios, Phys. Rev. C 90, 054327 (2014).

[15] L.-W. Chen, B.-J. Cai, C. M. Ko, B.-A. Li, C. Shen, and J. Xu, Phys. Rev. C 80, 014322 (2009).

[16] H.-Y. Kong, J. Xu, L.-W. Chen, B.-A. Li, and Y.-G. Ma, Phys. Rev. C 95, 034324 (2017).

[17] S. Yoshida and H. Sagawa, Phys. Rev. C 73, 044320 (2006).

[18] P. Danielewicz and J. Lee, Nucl. Phys. A 818, 36 (2009).

[19] I. Vidaña, C. Providência, A. Polls, and A. Rios, Phys. Rev. C 80, 045806 (2009).

[20] C. Ducoin, J. Margueron, C. Providência, and I. Vidaña, Phys. Rev. C 83, 045810 (2011).

[21] J. Dong, W. Zuo, J. Gu, and U. Lombardo, Phys. Rev. 
C 85, 034308 (2012).

[22] B. M. Santos, M. Dutra, O. Lourenço, and A. Delfino, Phys. Rev. C 90, 035203 (2014).

[23] K. A. Brueckner and D. T. Goldman, Phys. Rev. 116, 424 (1959).

[24] D. Bandyopadhyay, C. Samanta, S. K. Samaddar, and J. N. De, Nuclear Physics A 511, 1 (1990).

[25] N. Hugenholtz and L. van Hove, Physica 24, 363 (1958).

[26] R. Chen, B.-J. Cai, L.-W. Chen, B.-A. Li, X.-H. Li, and C. Xu, Phys. Rev. C 85, 024305 (2012).

[27] J. N. De, S. K. Samaddar, and B. K. Agrawal, Phys. Rev. C 92, 014304 (2015).

[28] A. Bohr and B. R. Mottelson, Nuclear Structure, vol. I (Benjamin, New York, 1969).

[29] A. Akmal, V. Pandharipande, and D. Ravenhall, Phys. Rev. C 58, 1804 (1998).

[30] G. Taranto, M. Baldo, and G. F. Burgio, Phys. Rev. C 87, 045803 (2013).

[31] M. Baldo, L. M. Robledo, P. Schuck, and X. Viñas, Phys. Rev. C 87, 064305 (2013).

[32] B. K. Agrawal, S. K. Samaddar, J. N. De, C. Mondal, and S. De, International Journal of Modern Physics E 26, 1750022 (2017).

[33] E. Khan, J. Margueron, and I. Vidaña, Phys. Rev. Lett. 109, 092501 (2012).

[34] B. A. Brown, Phys. Rev. Lett. 111, 232502 (2013).
[35] W. D. Myers and W. J. Swiatecki, Phys. Rev. C 57, 3020 (1998).

[36] W.D.Myers and W.J.Swiaetecki, Nucl. Phys. A601, 141 (1996).

[37] A. Akmal and V. R. Pandharipande, Phys. Rev. C 56, 2261 (1997).

[38] M. Jaminon and C. Mahaux, Phys. Rev. C 40, 354 (1989).

[39] X.-H. Li, W.-J. Guo, B.-A. Li, L.-W. Chen, F. J. Fattoyev, and W. G. Newton, Phys. Lett. B 743, 408 (2015).

[40] B.-A. Li and X. Han, Phys. Lett. B 727, 276 (2013).

[41] Z. Zhang and L.-W. Chen, Phys. Rev. C 90, 064317 (2014).

[42] T. Li and et al, Phys. Rev. Lett. 99, 162503 (2007).

[43] J. M. Pearson, N. Chamel, and S. Goriely, Phys. Rev. C 82, 037301 (2010).

[44] Z. Zhang and L.-W. Chen, Phys. Rev. C 93, 034335 (2016).

[45] Z. Zhang and L.-W. Chen, Physics Letters B 726, 234 (2013), ISSN 0370-2693.

[46] C. Li-Gang and M. Zhong-Yu, Chinese Physics Letters 25, 1625 (2008).

[47] M. Liu, N. Wang, Z.-X. Li, and F.-S. Zhang, Phys. Rev. C 82, 064306 (2010). 